Center for

Mathematical Economics

Working Papers

September 2014

\title{
A Dynamic Extension of the Foster-Hart Measure of Riskiness
}

Tobias Hellmann and Frank Riedel

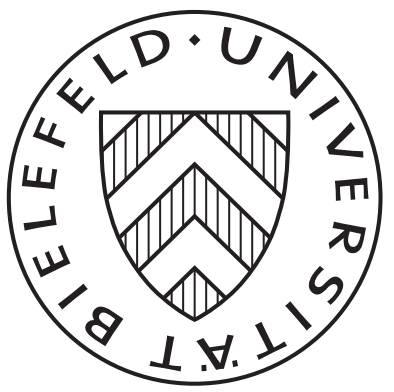

Center for Mathematical Economics (IMW)

Bielefeld University

Universitätsstraße 25

D-33615 Bielefeld · Germany

e-mail: imw@uni-bielefeld.de

http://www.imw.uni-bielefeld.de/wp/

ISSN: 0931-6558 


\title{
A Dynamic Extension of the Foster-Hart Measure of Riskiness
}

\author{
Tobias Hellmann * $\quad$ Frank Riedel ${ }^{\dagger}$
}

September 26, 2014

\begin{abstract}
We analyze the Foster-Hart measure of riskiness for general distributions in dynamic settings. The Foster-Hart measure avoids bankruptcy in the long run. It is not time-consistent.

Key words and phrases: Dynamic Risk Measures, Time-Consistency, Bankruptcy, Continuous Random Variable

JEL subject classification: D81, G11
\end{abstract}

${ }^{*}$ Center for Mathematical Economics (IMW) and Bielefeld Graduate School of Economics and Management (BiGSEM), Bielefeld University, tobias.hellmann@unibielefeld.de. Financial support provided by the International Research Training Group "Economic Behavior and Interaction Models" (EBIM) is gratefully acknowledged.

$\dagger$ 


\section{Introduction}

Foster and Hart (2009) introduce a notion of riskiness, or critical wealth level, for gambles with known distribution. Their measure is objective in so far as it depends only on the distribution of the outcome; in the language of the finance literature on risk measures, it is law-invariant, in decision-theoretic terms, it is probabilistically sophisticated.

Formally, the Foster-Hart measure of riskiness is given by the unique solution $R(X)$ of

$$
E \log \left(1+\frac{X}{R(X)}\right)=0 .
$$

This concept admits a simple operational interpretation as an agent avoids bankruptcy in the long run provided she accepts a gamble $X$ only if her wealth exceeds the critical value $R(X)$. The Foster-Hart measure of riskiness $R(X)$ is defined for a discrete random variable $X$ on some probability space $(\Omega, \mathcal{F}, P)$ that satisfies $E X>0$ and $P(X<0)>0$.

Hellmann and Riedel (2014) noticed that for continuous distributed gambles the defining equation does not necesseraly posses a solution. By showing that the riskiness numbers of discrete gambles on a grid converge to the maximal loss of these gambles as the grid size becomes finer, they suggest to use the maximal loss as the reasonable extension for the Foster-Hart measure in case there exists no solution to equation (1).

Until now the Foster-Hart measure of riskiness has just been studied in a static framework. As many financial applications require, however, to quantify risk over time in a dynamic way, it seems natural and important to generalize the concept to a dynamic framework.

Dynamic measurement of risk plays an important role in the recent literature ${ }^{1}$ since it allows, in contrast to the static case, to measure risk of financial positions over time. The arrival of new information can thus be taken into account. This is important for many situations; suppose, for instance, one faces a gamble that has its payments in, say, one month. In two weeks from now the information about this gamble might be much more precise which allows to adjust the risk assessment and to determine the risk more accurately. A static risk measure cannot do that. To cover such cases it is therefore crucial to be able to merge from static to dynamic risk measurement.

\footnotetext{
${ }^{1}$ See, among others, Detlefsen and Scandolo (2005) and Föllmer and Schied (2011), Chapter 11 for a detailed introduction to dynamic risk measures.
} 
We thus set out to study the Foster-Hart measure of riskiness (or more precisely the extended Foster-Hart measure of riskiness defined in Hellmann and Riedel (2014)) in a dynamic framework. We define the dynamic extended Foster-Hart measure of riskiness.

A desirable property of a dynamic risk measure is the notion of timeconsistency. Roughly speaking a measure is time consistent if it assigns a greater risk to one gamble than to another whenever it is known that the same holds true tomorrow. This property yields a consistent behavior of an agent who bases her decision on a time consistent risk measure. We show, however, that the dynamic version of the Foster-Hart measure of riskiness does not satisfy the time-consistency condition.

We further give an important justification for the extended Foster-Hart measure of riskiness by showing that the no-bankruptcy result (and with it the operational interpretation) carries over to continuous distributions.

In the original work of Foster and Hart (2009) a somewhat dynamic approach is already needed to prove the no-bankruptcy result. Their approach, however, is rather intuitive than measure theoretically precise. We provide here a precise measure theoretic approach, which allows us also to drop the quite artificial assumption used in Foster and Hart (2009) that all gambles are multiples of a finite number of basic gambles.

Furthermore, we have to cope with the fact that for the extended FosterHart measure of riskiness it is allowed, in contrast to original measure, that the possible gains are unbounded. We therefore deal with a more general class of gambles.

For all these reasons we come up with a completely different approach to prove the no-bankruptcy result.

The paper is set up as follows: Section 2 introduces the dynamic framework as well as the dynamic extended Foster-Hart measure of riskiness. In Section 3 we give the more general no-bankruptcy result. Section 4 contains a counterexample which shows the time-inconsistency of the new defined measure. Finally, the appendix consists of some proofs.

\section{The dynamic framework}

In the following, let $\left(\Omega, \mathcal{F},\left(\mathcal{F}_{t}\right)_{t \in \mathbb{N}}, P\right)$ be a filtered probability space, where the filtration $\left(\mathcal{F}_{t}\right)_{t \in \mathbb{N}}$ represents the information structure given at the respective time $t$. We denote by $\mathcal{A}_{t}$ the set of all $\mathcal{F}_{t}-$ measurable random variables 
and consider a sequence of random variables $\left(X_{t}\right)$ that is adapted to the filtration $\left(\mathcal{F}_{t}\right)_{t \in \mathbb{N}}$. In order to be able to measure the risk of $X_{t}$ in every time period $s<t, X_{t}$ has to satisfy all the conditions which define a gamble in Hellmann and Riedel (2014) given the respective information structure $\left(\mathcal{F}_{s}\right)$.

Definition 2.1 We call a random variable $X$ on $(\Omega, \mathcal{F}, P)$ a gamble for the $\sigma$-field $\mathcal{F}_{s} \subset \mathcal{F}$ if $X$ is bounded from below and satisfies $E\left[X \mid \mathcal{F}_{s}\right]>0$ a.s. and $P\left(X_{t}<0 \mid \mathcal{F}_{s}\right)>0$ a.s..

In the remainder, we assume that for $t>s, X_{t}$ is a gamble for $\mathcal{F}_{s}$. We denote by $L_{s}\left(X_{t}\right)$ the maximal loss of $X_{t}$ given the information at time $s$. Formally,

$$
L_{s}\left(X_{t}\right):=\operatorname{ess} \inf \left\{Z \in \mathcal{A}_{s} \mid P\left(-X_{t}>Z \mid \mathcal{F}_{s}\right)=0 \text { a.s. }\right\} .
$$

We now embed the extended riskiness notion of Hellmann and Riedel (2014) in the dynamic framework. As time goes by, we learn something about the realization of the random variable and are therefore able to quantify the risk more precisely. Measuring the risk of $X_{t}$ in every single time period $s<t$ yields a family of conditional risk measures $\left(\rho_{s}\left(X_{t}\right)\right)_{s=1 \ldots t-1}$, where every $\rho_{s}\left(X_{t}\right)$ is a $\mathcal{F}_{s}$-measurable random variable. For continuous random variables the equation

$$
E\left[\log \left(1+\frac{X_{t}}{\rho_{s}\left(X_{t}\right)}\right) \mid \mathcal{F}_{s}\right]=0
$$

does not always have a solution. Following the arguments of Hellmann and Riedel (2014), this is the case on the set

$$
\mathcal{B}:=\left\{E\left[\log \left(1+\frac{X_{t}}{L_{s}\left(X_{t}\right)}\right) \mid \mathcal{F}_{s}\right] \geq 0\right\} .
$$

As in the static case, on $\mathcal{B}$ the conditional maximal loss is the reasonable extension of the classical riskiness notion. We are now ready to give the definition of the dynamic extended Foster-Hart measure of riskiness.

Definition 2.2 The dynamic extended Foster-Hart measure of riskiness for a gamble $X_{t}$ is the family of conditional risk measures $\left(\rho_{s}\left(X_{t}\right)\right)_{s=1 \ldots .-1}$, where each $\rho_{s}\left(X_{t}\right)$ is equal to the conditional maximal loss $L_{s}\left(X_{t}\right)$ on $\mathcal{B}$ and the solution to equation (2) on $\mathcal{B}^{c}$. 
The next lemma shows that the dynamic extended Foster-Hart riskiness is indeed well defined.

Lemma 2.3 There exists one and only one $\mathcal{F}_{s}$-measurable random variable $\rho_{s}\left(X_{t}\right) \geq L_{s}\left(X_{t}\right)$ that solves equation $(2)$ on $\mathcal{B}^{c}$ and satisfies $\rho_{s}\left(X_{t}\right)=L_{s}\left(X_{t}\right)$ on $\mathcal{B}$.

For the proof we refer to the appendix.

\section{No-Bankruptcy Result}

The main result of Foster and Hart (2009) yields that a decision maker who rejects a gamble whenever his wealth is below the associated riskiness number avoids bankruptcy (with probability one). It is crucial not to lose this property (and with it the operational interpretation of the measure) when working with continuous distributed gambles.

We provide here the respective no-bankruptcy theorem for the extended Foster-Hart measure of riskiness.

Theorem 3.1 Let $\left(X_{n}\right)$ be a sequence of gambles that are uniformly bounded above by some integrable random variable $Y>0$ and satisfy some minimal possible loss requirement, i.e. there exists $\epsilon>0$ such that a.s.

$$
L_{n-1}\left(X_{n}\right) \geq \epsilon>0
$$

for all $n$. Let $W_{0}>0$ be the initial wealth and define recursively

$$
W_{t+1}=W_{t}+X_{t+1}
$$

if $E\left[\log \left(1+\frac{X_{t+1}}{W_{t}}\right) \mid \mathcal{F}_{t}\right] \geq 0$ and

$$
W_{t+1}=W_{t}
$$

else. We then ensure no bankruptcy, i.e.

$$
P\left[\lim W_{t}=0\right]=0 .
$$

Proof: Note first that $W_{t}>0$. This can be shown by induction. We have $W_{0}>0$. We have either $W_{t+1}=W_{t}$ which is positive by induction hypothesis, 
or $W_{t+1}=W_{t}+X_{t+1}$. In this case, the condition $E\left[\log \left(1+\frac{X_{t+1}}{W_{t}}\right) \mid \mathcal{F}_{t}\right] \geq 0$ implies that

$$
W_{t} \geq \rho_{t}\left(X_{t+1}\right) \geq L_{t}\left(X_{t+1}\right) .
$$

Thus, $W_{t}-L_{t}\left(X_{t+1}\right) \geq 0$. If $\rho_{t}\left(X_{t+1}\right)=L_{t}\left(X_{t+1}\right)$, then we have $P\left(X_{t+1}=\right.$ $\left.L_{t}\left(X_{t+1}\right) \mid \mathcal{F}_{t}\right)=0$. Hence, it holds that

$$
W_{t+1}>W_{t}-L_{t}\left(X_{t+1}\right) \geq 0 .
$$

We can thus define $S_{t}=\log W_{t}$. We claim that $S$ is a submartingale. Indeed, on the set

$$
A:=\left\{E\left[\log \left(1+\frac{X_{t+1}}{W_{t}}\right) \mid \mathcal{F}_{t}\right]<0\right\}
$$

which belongs to $\mathcal{F}_{t}$, there is nothing to show. On the set $A^{c}$, we have

$$
\begin{aligned}
E\left[S_{t+1} \mid \mathcal{F}_{t}\right] & =E\left[\log W_{t+1} \mid \mathcal{F}_{t}\right] \\
& =\log W_{t}+E\left[\log \frac{W_{t+1}}{W_{t}} \mid \mathcal{F}_{t}\right] \\
& =\log W_{t}+E\left[\log \left(1+\frac{X_{t+1}}{W_{t}}\right) \mid \mathcal{F}_{t}\right] \\
& \geq \log W_{t}=S_{t} .
\end{aligned}
$$

$S$ is thus a submartingale. We apply the theorem on submartingale convergence in Shiryaev (1984), Chapter VII, Theorem 1. For $a>0$, let $\tau_{a}=\inf \left\{t \geq o: X_{t}>a\right\}$. A stochastic sequence belongs to class $\mathcal{C}^{+}$if for every $a>0$ we have

$$
E\left(X_{\tau_{a}}-X_{\tau_{a}-1}\right)^{+} 1_{\left\{\tau_{a}<\infty\right\}}<\infty .
$$

Let us check that our sequence $S$ is of class $\mathcal{C}^{+}$. Indeed, we have

$$
\left(S_{\tau_{a}}-S_{\tau_{a}-1}\right)^{+}=\log \left(1+\frac{X_{\tau_{a}}}{W_{\tau_{a}-1}}\right) 1_{\left\{X_{\tau_{a}} \geq 0\right\}}
$$

and in that case $W_{\tau_{a}-1} \geq \rho_{\tau_{a}-1}\left(X_{\tau_{a}}\right) \geq \epsilon>0$, so we conclude

$$
E\left(S_{\tau_{a}}-S_{\tau_{a}-1}\right)^{+} \leq E \log \left(1+\frac{Y}{\rho_{\tau_{a}-1}\left(X_{\tau_{a}}\right)}\right) \leq E \log \left(1+\frac{Y}{\epsilon}\right) \leq E \frac{Y}{\epsilon}<\infty
$$

where $Y$ is the uniform integrable upper bound for our gambles and $\epsilon$ is the minimal possible loss lower bound. By Theorem 1 in Shiryaev (1984), 
Chapter VII, we conclude that the set $\left\{S_{t} \rightarrow-\infty\right\}$ is a null set. Indeed, on the set $\left\{S_{t} \rightarrow-\infty\right\}, S$ is bounded above. The theorem then states that the limit of $S$ exists and is finite (almost surely), and thus cannot be negative infinity.

\section{Time Consistency}

An important question arising in a dynamic framework is how the conditional risks at different times are interrelated. This question leads to the important notion of time-consistency. A dynamic risk measure $\left(\rho_{s}\right)_{s=1 \ldots t-1}$ is called time-consistent if for any gamble $X_{t}^{1}, X_{t}^{2}$ and for all $s=1, \ldots, t-2$ it holds that

$$
\rho_{s+1}\left(X_{t}^{1}\right) \geq \rho_{s+1}\left(X_{t}^{2}\right) \text { a.s. } \Longrightarrow \rho_{s}\left(X_{t}^{1}\right) \geq \rho_{s}\left(X_{t}^{2}\right) \text { a.s. }
$$

That means, in particular, that if we know that tomorrow in every state of the world gamble $X_{t}^{1}$ is assigned to have a higher risk than gamble $X_{t}^{2}$, this should also hold true today.

This desirable property is not satisfied by the dynamic extended FosterHart riskiness as the following example demonstrates.

Example 4.1 Consider two discrete gambles $X_{2}^{1}$ and $X_{2}^{2}$ that have their payments in two periods $(t=2)$ from now. They are distributed according to the binomial trees given below. In $t=1$ two states of the world are possible which occur with equal probability $\frac{1}{2}$. We compute the riskiness today $(t=0)$ and in each state in $t=1$.

Gamble $X_{2}^{1}$ has the following structure:

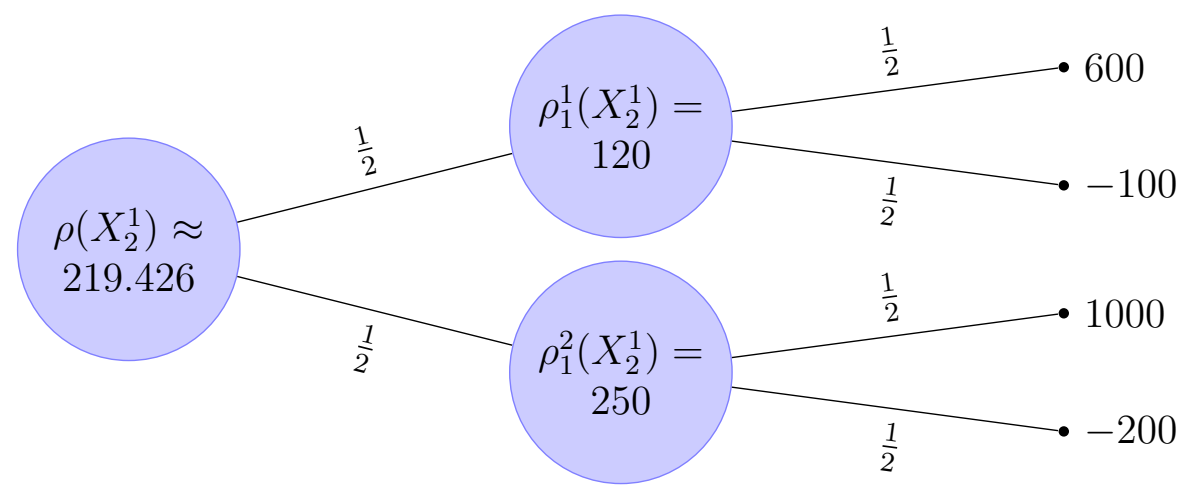


Hence, $X_{2}^{1}$ has the payoffs $\{600,-100,1000,-200\}$ occurring each with equal probability. The riskiness in $t=1$ in state one is the unique positive solution to the equation $1 / 2 \log \left(1+600 / \rho_{1}^{1}\left(X_{2}^{1}\right)\right)+1 / 2 \log \left(1-100 / \rho_{1}^{1}\left(X_{2}^{1}\right)\right)=$ 0 . Solving this gives $\rho_{1}^{1}\left(X_{2}^{1}\right)=120$. In a similar way we get that the riskiness number in state two is $\rho_{1}^{2}\left(X_{2}^{1}\right)=250$ and that the riskiness today is $\rho\left(X_{2}^{1}\right) \approx$ 219.426.

The second gamble $X_{2}^{2}$ is distributed according to the following tree:

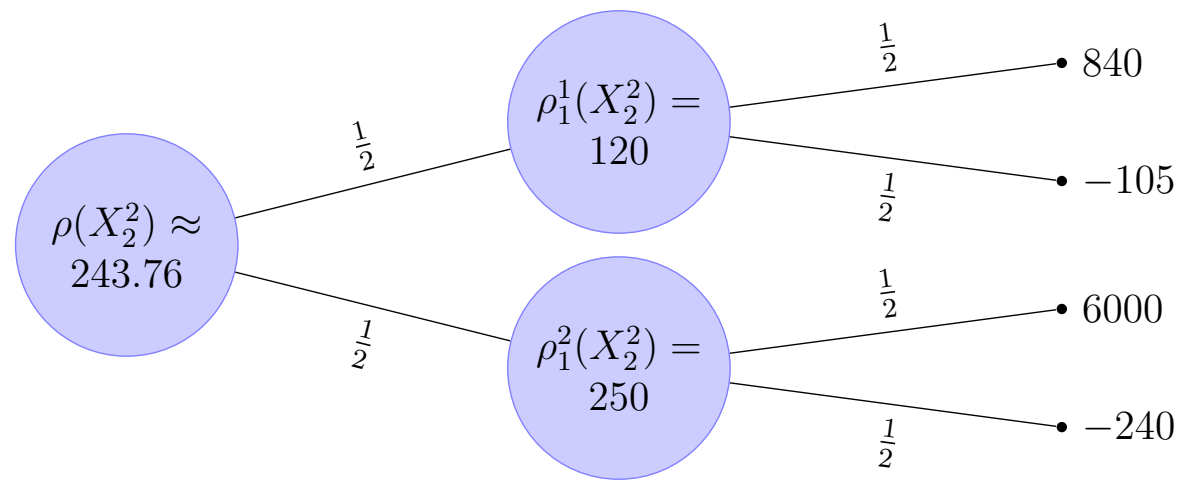

Although the payoffs of $X_{2}^{2}$ differ from the payoffs of $X_{2}^{1}$, the riskiness numbers at time $t=1$ coincides. Today, however, the risk of $X_{2}^{2}$ is strictly greater than the risk of $X_{2}^{1}$ which contradicts the time-consistency condition (3). Therefore, the dynamic extended Foster-Hart riskiness is not timeconsistent.

\section{Appendix}

For the appendix all inequalities and equalities between random variables are assumed to hold $P$-almost surely.

\section{A Proof of Lemma 2.3}

Proof of Lemma 2.3: Let $X_{t}$ be a gamble for the $\sigma$-field $\mathcal{F}_{s}$. Without loss of generality, we can assume $L_{s}\left(X_{t}\right)=1$ almost surely (else replace $X_{t}$ by $\left.X_{t} / L_{s}\left(X_{t}\right)\right)$.

We write $\mathcal{G}=\mathcal{F}_{s}$ and $X=X_{t}$ in the following for shorter notation. We fix a regular version $P(\omega, d x)$ for the conditional probability distribution of $X$ given $\mathcal{G}$ (which exists as $X$ takes values in a Polish space). Whenever we 
write conditional expectations or probabilities in the following, we have this regular version in mind.

For our construction, we need that there are wealth levels $W$ for which we accept the gamble $X$ given $\mathcal{G}$. We thus start with the following observation.

Lemma A.1 There exist $\mathcal{G}-$ measurable random variables $W \geq 1$ such that

$$
E[\log (W+X) \mid \mathcal{G}] \geq \log W .
$$

In particular, this holds true for all $W$ with

$$
W \geq \frac{2 E\left[X^{2} \mid \mathcal{G}\right]}{E[X \mid \mathcal{G}]}
$$

and

$$
\left|\frac{X}{W}\right| \leq \frac{1}{2}
$$

Proof of Lemma A.1: We use the estimate

$$
\log (1+x) \geq x-2 x^{2}
$$

for $|x| \leq 1 / 2$ (which one can obtain from a Taylor-expansion and the Lagrange version of the error term). Take an $\mathcal{G}$-measurable $W$ with

$$
W \geq \frac{2 E\left[X^{2} \mid \mathcal{G}\right]}{E[X \mid \mathcal{G}]}
$$

and

$$
\left|\frac{X}{W}\right| \leq \frac{1}{2}
$$

Such $W$ exists because $X$ has finite variance. We can simply take

$$
W=2 \max \left\{\frac{E\left[X^{2} \mid \mathcal{G}\right]}{E[X \mid \mathcal{G}]}, 2|X|\right\} .
$$

As $|X / W| \leq 1 / 2, \log (1+X / W)$ is everywhere defined. By the estimate (4), we obtain

$$
\begin{aligned}
E\left[\log \left(1+\frac{X}{W}\right) \mid \mathcal{G}\right] & \geq E\left[\frac{X}{W}-\frac{2 X^{2}}{W^{2}} \mid \mathcal{G}\right] \\
& =\frac{1}{W}\left(E[X \mid \mathcal{G}]-\frac{E\left[2 X^{2} \mid \mathcal{G}\right]}{W}\right)
\end{aligned}
$$


and now we can use the fact that $W \geq \frac{2 E\left[X^{2} \mid \mathcal{G}\right]}{E[X \mid \mathcal{G}]}$ to conclude that

$$
E\left[\log \left(1+\frac{X}{W}\right) \mid \mathcal{G}\right] \geq 0 .
$$

As a consequence of the preceding lemma, the set

$$
\Lambda=\{\lambda \mathcal{G} \text { - measurable } 0<\lambda \leq 1, E[\log (1+\lambda X) \mid \mathcal{G}] \geq 0\}
$$

is not empty. Let $\lambda_{0}$ be the $\mathcal{G}$-essential supremum of $\lambda$. By definition, $\lambda_{0}$ is $\mathcal{G}$-measurable and $\lambda_{0} \geq \lambda$ for all $\lambda \in \Lambda$. Moreover, $\lambda_{0}$ is the smallest $\mathcal{G}$-measurable random variable with these properties.

The set $\Lambda$ is upwards directed: take $\lambda_{1}, \lambda_{2} \in \Lambda$. Then we have for $\lambda_{3}=$ $\max \left\{\lambda_{1}, \lambda_{2}\right\}$

$$
\begin{aligned}
E\left[\log \left(1+\lambda_{3} X\right) \mid \mathcal{G}\right] & =1_{\left\{\lambda_{1} \geq \lambda_{2}\right\}} E\left[\log \left(1+\lambda_{1} X\right) \mid \mathcal{G}\right] \\
& +1_{\left\{\lambda_{1}<\lambda_{2}\right\}} E\left[\log \left(1+\lambda_{2} X\right) \mid \mathcal{G}\right] \geq 0 .
\end{aligned}
$$

The other properties being obvious, we conclude $\lambda_{3} \in \Lambda$. Hence, $\Lambda$ is upwards directed; as a consequence, there exists a sequence $\left(\lambda_{n}\right) \subset \Lambda$ with $\lambda_{n} \uparrow \lambda_{0}$.

Our next claim is $E\left[\log \left(1+\lambda_{0} X\right) \mid \mathcal{G}\right] \geq 0$. The sequence

$$
Z_{n}=-\log \left(1+\lambda_{n} X\right)
$$

is bounded from below by $-\log (1+|X|) \geq-|X| \in L^{1}$. We can then apply Fatou's lemma to conclude

$$
-E\left[\log \left(1+\lambda_{0} X\right) \mid \mathcal{G}\right]=E\left[\lim Z_{n} \mid \mathcal{G}\right] \leq \liminf -E\left[\log \left(1+\lambda_{n} X_{n}\right) \mid \mathcal{G}\right] \leq 0,
$$

or

$$
E\left[\log \left(1+\lambda_{0} X\right) \mid \mathcal{G}\right] \geq 0 .
$$

We claim now that we have

$$
E\left[\log \left(1+\lambda_{0} X\right) \mid \mathcal{G}\right]=0
$$

on the set $\left\{\lambda_{0}<1\right\}$. This will conclude the proof of our lemma.

It is enough to establish the claim on all sets

$$
\Gamma_{n}=\left\{\lambda_{0} \leq 1-\frac{1}{n}\right\}
$$


for all $n \in \mathbb{N}$. From now on, we work on this set only without stating it explicitly. Let

$$
A_{m, n}=\left\{E\left[\log \left(1+\lambda_{0} X\right) \mid \mathcal{G}\right] \geq \frac{1}{m}\right\} \cap \Gamma_{n} .
$$

We will show that $A_{m, n}$ is a null set for all $m, n \in \mathbb{N}$.

Let $\epsilon=\frac{1}{1+m n}$ and set $\lambda_{1}=(1-\epsilon) \lambda_{0}+\epsilon$. Then we have $\lambda_{1}>\lambda_{0}$ and $\lambda_{1} \leq(1-\epsilon)(1-1 / n)+\epsilon=1-1 / n+\epsilon / n<1$. We also note

$$
\lambda_{1}-\lambda_{0}=\epsilon\left(1-\lambda_{0}\right) \leq \epsilon
$$

We have

$$
1+\lambda_{1} X \geq 1-\lambda_{1} \geq \frac{1-\epsilon}{n}>0 .
$$

$\log \left(1+\lambda_{1} X\right)$ is thus finite (on $\Gamma_{n}$ where we work).

We now want to show

$$
E\left[\log \left(1+\lambda_{1} X\right) \mid \mathcal{G}\right] \geq 0
$$

on $A_{m, n}$. If $A_{m, n}$ was not a null set, this would contradict the definition of $\lambda_{0}$ as the $\mathcal{G}$-essential supremum of $\Lambda$.

In order to establish the desired inequality, it is enough to show

$$
E\left[\log \left(1+\lambda_{1} X\right) \mid \mathcal{G}\right]-E\left[\log \left(1+\lambda_{0} X\right) \mid \mathcal{G}\right] \geq-\frac{1}{m}
$$

because of the definition of $A_{m, n}$. Now, on the set $\{X \geq 0\}$ we have $\log \left(1+\lambda_{1} X\right) \geq \log \left(1+\lambda_{0} X\right)$.

We need a uniform estimate for $\log \left(1+\lambda_{1} X\right)-\log \left(1+\lambda_{0} X\right)$ on the set $\{X<0\}$. With the help of the mean value theorem, we obtain on $\{X<0\}$

$$
\log \left(\frac{1+\lambda_{1} X}{1+\lambda_{0} X}\right) \geq-\frac{n}{1-\epsilon}\left(\lambda_{1}-\lambda_{0}\right) \geq-\frac{n \epsilon}{1-\epsilon}
$$

(How can one see this: by the mean value theorem and (6), we have

$$
\log \left(1+\lambda_{1} X\right)-\log \left(1+\lambda_{0} X\right)=\frac{1}{\xi}\left(\lambda_{1}-\lambda_{0}\right) X
$$

for some $\xi$ in between $1+\lambda_{1} X$ and $1+\lambda_{0} X$. By (7) $1+\lambda_{1} X \geq(1-\epsilon) / n$. Hence, we have $0<1 / \xi \leq \frac{n}{1-\epsilon}$.) 
By the definition of $\epsilon$, we thus have

$$
\log \left(\frac{1+\lambda_{1} X}{1+\lambda_{0} X}\right) \geq-\frac{n \epsilon}{1-\epsilon}=-\frac{1}{m}
$$

uniformly on $\{X<0\}$ as desired. It follows

$$
\begin{aligned}
& E\left[\log \left(1+\lambda_{1} X\right) \mid \mathcal{G}\right]-E\left[\log \left(1+\lambda_{0} X\right) \mid \mathcal{G}\right] \\
& \geq E\left[\log \left(\frac{1+\lambda_{1} X}{1+\lambda_{0} X}\right) 1_{\{X<0\}} \mid \mathcal{G}\right] \\
& \geq-\frac{1}{m} .
\end{aligned}
$$

\section{References}

Detlefsen, K. and Scandolo, G. (2005). Conditional and dynamic convex risk measures. Finance and Stochastics, 9(4):539-561.

Föllmer, H. and Schied, A. (2011). Stochastic finance. An introduction in discrete time. de Gruyter, Berlin [u.a.].

Foster, D. and Hart, S. (2009). An operational measure of riskiness. Journal of Political Economy, 117:785-814.

Hellmann, T. and Riedel, F. (2014). The foster-hart measure of riskiness for general gambles. Theoretical Economics, to appear.

Shiryaev, A. N. (1984). Probability. Graduate texts in mathematics ; 95. Springer. 